\title{
Intrinsically magnetic susceptibility in human blood and its impact on cell separation: Non-classical and intermediate monocytes have the strongest magnetic behavior in fresh human blood
}

Jenifer Gómez-Pastora ${ }^{1}$, James Kim ${ }^{1}$, Victor Multanen ${ }^{1}$, Mitchell Weigand ${ }^{1}$, Nicole Walters $^{1}$, Eduardo Reátegui ${ }^{1}$, Andre Palmer ${ }^{2}$, Mark Yazer ${ }^{3}$, Maciej Zborowski ${ }^{4}$, and Jeffrey Chalmers ${ }^{1}$

${ }^{1}$ The Ohio State University

${ }^{2}$ the Ohio State University

${ }^{3}$ University of Pittsburg

${ }^{4}$ Cleveland Clinic

January 19, 2021

\begin{abstract}
The presence of iron in circulating monocytes is well known as they play an essential role in iron recycling. It has been demonstrated that the iron content of blood cells can be measured through their magnetic behavior; however, the magnetic properties of different monocyte subtypes remain unknown. In this study, we report for the first time, the magnetic behavior of classical, intermediate and non-classical monocytes, which is related to their iron storage capacity. The magnetic properties of monocytes were compared to other blood cells, such as lymphocytes and red blood cells in the oxyhemoglobin and methemoglobin states, and a cancer cell type. For this analysis, we used an instrument referred to as Cell Tracking Velocimetry (CTV), which quantitatively characterizes the magnetic behavior of biological entities. Our results demonstrate that significant fractions of the intermediate and non-classical monocytes have high magnetophoretic mobilities, equivalent to methemoglobin red blood cells and higher than the classical subset, suggesting their higher iron storage capacities. Moreover, our findings have implications for the immunomagnetic separation industry; we demonstrate that negative magnetic isolation techniques for recovering monocytes from blood should be used with caution, as it is possible to lose magnetic monocytes when using this technique.
\end{abstract}

\section{Hosted file}

Text.pdf available at https://authorea.com/users/367359/articles/504806-intrinsicallymagnetic-susceptibility-in-human-blood-and-its-impact-on-cell-separation-non-classicaland-intermediate-monocytes-have-the-strongest-magnetic-behavior-in-fresh-human-blood

\section{Hosted file}

Figures.pdf available at https://authorea.com/users/367359/articles/504806-intrinsicallymagnetic-susceptibility-in-human-blood-and-its-impact-on-cell-separation-non-classicaland-intermediate-monocytes-have-the-strongest-magnetic-behavior-in-fresh-human-blood 